\title{
FLUORESCENCE YIELD NEAR EDGE SPECTROSCOPY OF $\pi$-BONDED CO ON Fe(100)
}

\section{D.J. DWYER *, B. RAUSENBERGER}

Department of Chemistry, Laboraton for Surface Science and Technology, University of Maine, Orono, $M E$ 04469, USA

\section{J.P. LU, S.L. BERNASEK}

Department of Chemistry, Princeton University, Princeton, NJ 08544, USA

\section{D.A. FISCHER}

Exxon PRT, Brookhaven National Laboratory, Building $510 E$, Upton, NY, USA

\section{S.D. CAMERON}

Exxon Research and Engineering, Analytical Science Laboratory, Route 22 East, Annandale, NJ 08801, USA

\section{D.H. PARKER}

Department of Chemistry, University of Colorado at Boulder, Boulder, CO 80309, USA

and

\section{J.L. GLAND}

Department of Chemistry, University of Michigan, Ann Arbor, MI 48109, USA

Received 28 March 1989; accepted for publication 30 June 1989

Near edge X-ray absorption fine structure (NEXAFS) spectra of CO adsorbed on the $\mathrm{Fe}(100)$ surface are reported. Spectra, obtained by fluorescence yield (FYNES), are presented for each of the four individual $\mathrm{CO}$ adsorption configurations observed on this surface. The $\pi$-bonded state exhibits an unusual FYNES spectrum and polarization dependence which indicates that the molecule is either extensively rehybridized or tilted with respect to the surface normal. The FYNES spectra of each of the adsorption states directly reflect the perturbation of the carbon-oxygen bond by the surface and track systematically with the heat of adsorption.

* To whom correspondence should be addressed. 


\section{Introduction}

The adsorption and dissociation of $\mathrm{CO}$ on $\mathrm{Fe}(100)$ is of particular interest due to the isolation of a precursor state to $\mathrm{CO}$ dissociation [1]. A rich history of experimental work has resulted in a gradual evolution of our understanding of the adsorption/dissociation process on this surface. Early photoemission studies focused on the issue of whether $\mathrm{CO}$ adsorption was molecular or dissociative [2-4]. Later studies showed that the adsorption process is complex with at least three different molecular adsorption states coexisting on the surface at cryogenic temperatures. Dissociation occurs from a single molecular state at approximately $440 \mathrm{~K}[5,6]$. This precursor state to dissociation is the first to fill and saturate, at adsorption temperatures below $300 \mathrm{~K}$. with the other two molecular states filling sequentially. A surface which contains only the precursor state can be prepared by carefully controlling the $\mathrm{CO}$ dose or by annealing a $\mathrm{CO}$ saturated surface at $350 \mathrm{~K}$. One engaging aspect of this precursor state is its unusually low carbon-oxygen stretching frequency $(1200$ $\mathrm{cm}^{-1}$ ) as determined by EELS [7.8] and the large apparent tilt of the molecular axis with respect to the surface normal as determined NEXAFS [9]. These results coupled with subsequent UPS measurements [6.10] has lead to the speculation that the $\mathrm{CO}$ molecule is $\pi$-bonded to the surface in a lashion similar to various organometallic carbonyl clusters [11].

A detailed orbital description of the $\pi$-bonded state is not available hut there has been theoretical justification for parallel bonding of $\mathrm{CO}$ to the Fe(100) surface [12]. However, there is not good agreement between the orhital energies predicted by the theoretical study and the binding energies of bonding orbitals as measured by UPS.

In this study we continue to examine the chemical nature of the $\pi$-bonded state (we use the labels $\pi$-bonded, precursor and $\alpha_{3}-\mathrm{CO}$ interchangeably in this paper to describe $\mathrm{CO}$ bound to the surface in the pre-dissociated form) and report near-edge $X$-ray absorption spectra obtained via fluorescence yield measurements (fluorescence yield near-edge spectroscopy FYNES). These data were collected to complement previously collected NEXAFS data obtained by electron yield [9]. The earlier NEXAFS data were limited to only the (O) saturated surface (mixed molecular adsorption states) and the $\pi$-bonded state. Here we present FYNES spectra for the $\mathrm{CO} / \mathrm{Fe}(100)$ system as a function of various thermal treatments which allow us to extract the near-edge spectrat of each of the individual adsorption states on the surface. We were able to extract these data, in part, due to the superior signal to hackground of the fluorescence yield technique [13].

\section{Experimental}

Tunable radiation in the $275-350 \mathrm{eV}$ range was supplied by the extended range grasshopper monochromator (ERG) on the U1 beamline at the National 
Synchrotron Light Source, Brookhaven National Laboratory. The data were collected in a specialized UHV system designed to perform in-situ FYNES measurements in gaseous environments relevant to heterogeneous catalysis. This UHV system and the method of fluorescence detection has been described in detail elsewhere $[14,15]$. The Fe( 100$)$ crystal was mounted such that the electric field vector of the light could be rotated in the plane defined by the surface normal and the [110] direction. Unfortunately, the manipulator was not capable of azimuthal rotation.

The sample was cooled by $\mathrm{LN}_{2}$, heated resistively and the tempcraturc monitored by a type $\mathrm{K}$ thermocouple spot-welded to the back edge of the crystal. The temperature was accurately controlled by a programmer in the range $100-1000 \mathrm{~K}$. Sample cleaning procedures were the standard heating and sputtering cycles used to clean iron [1]. Sample cleanliness was verified by AES and temperature programmed CO desorption (CO TPD is extremely sensitive to surface impurities).

Since the collection of the FYNES data required an average of $20 \mathrm{~min}$ of data acquisition for each spectrum and since each experiment consisted of five such spectra, concern was raised as to the stability of the surface under the prolonged exposure to the synchrotron radiation. However, we were able to verify the stability of the surface by periodically performing a TPD experiment on an irradiated surface. There was no evidence of radiation damage (such as premature dissociation) observed in these experiments. We are confident that the prolonged data acquisition time did not influence the results of this study.

\section{Results}

The temperature programmed desorption of $\mathrm{CO}$ from the $\mathrm{Fe}(100)$ surface is shown in fig. 1. Four major desorption features are evident. The three features labeled $\alpha_{1}, \alpha_{2}$ and $\alpha_{3}$ are due to desorption of $\mathrm{CO}$ bound in molecular adsorption states, i.e. the carbon-oxygen bonds remain intact upon adsorption. The $\beta$ peak $(800 \mathrm{~K})$ is due to the second-order recombination of adsorbed atomic carbon and oxygen (dissociated $\mathrm{CO}$ ). These assignments have been verified by XPS, UPS [6], and EELS investigations [1]. The peak labeled $\alpha_{3}$ is the desorption feature associated with the $\pi$-bonded CO state.

The three molecular states are sequentially filled in the order of decreasing heat of adsorption and coexist on the surface at temperatures below $240 \mathrm{~K}$ $[1,6]$. The molecular states are sequentially desorbed in the reverse order during the TPD experiment. Heating the $\mathrm{CO}$ saturated surface to $240 \mathrm{~K}$ removes the $\alpha_{1}$ and heating to $350 \mathrm{~K}$ removes the $\alpha_{2}$ state. Heating the sample above $440 \mathrm{~K}$ results in a partial decomposition of the $\pi$-bonded $\alpha_{3}$ state as well as some desorption. The partitioning of the desorption versus dissociation channel has been discussed elsewhere [6]. A key feature of the desorption 


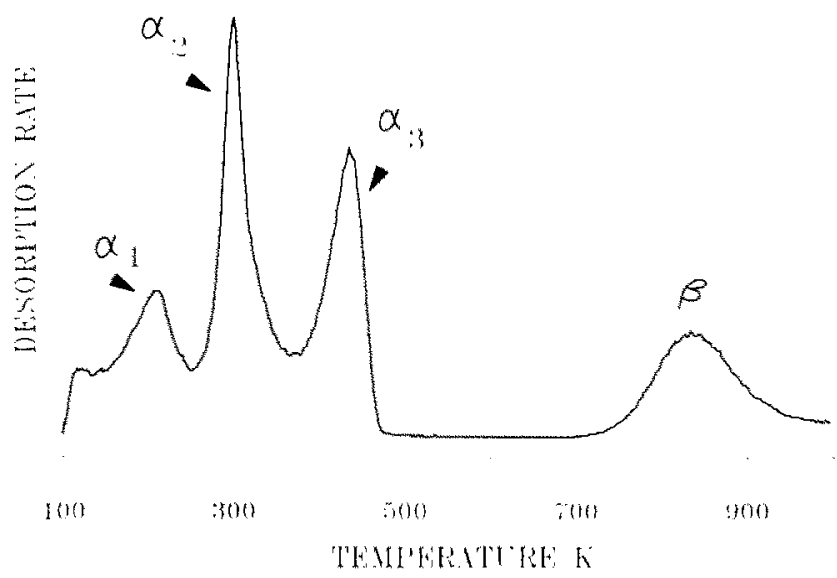

Fig. 1. Temperature programmed desorption spectrum of (O) from a $(\mathrm{C}$ saturated Fe(loo) surface. Heating ramp was $10 \mathrm{~K} / \mathrm{s}$ and the adsorption temperature was $8.3 \mathrm{~K}$.

experiment is that no $\beta$-CO exits on the surface prior to the $\mathrm{CO} \alpha_{3}$ desorption event. It is possible, to produce surfaces of mixed or pure $\mathrm{CO}$ adsorption states from the saturated surface by annealing at the correct temperature. For example, annealing a $\mathrm{CO}$ saturated surface at $350 \mathrm{~K}$ leads to a surface that contains only molecules bound in the $\pi$-bonded state.

The issue of surface coverage should be mentioned at this time. The ( () saturated surface coverage is $1.2 \times 10^{15}$ molecules $/ \mathrm{cm}^{2}$. one molecule per surface site $\left(\theta_{c,}=1\right)$. The sum of the $\alpha_{1}$ and $\alpha_{2}$ states is half a monolayer and the $\alpha_{3}$ state also occupies half a monolayer. The partitioning that occurs during the decomposition of the $\alpha_{3}$ state results in a quarter of a monolayer of $\beta-\operatorname{CO}\left(\theta_{0}-0.25, \theta_{0}-0.25\right)$. Both the $\alpha_{3}$ and $\beta-C O$ states form well defined $\mathrm{c}(2 \times 2)$ surface structures. The partitioning of the desorption versus decomposition channel appears to be the result of competition for the four-fold hollow sites on the surface [6].

Carbon edge FYNES data were obtained for $\mathrm{CO}$ adsorbed on the Fe(100) surface as a function of decreasing surface coverage in a set of annealing experiments. The results are shown for one angle of incidence $\left(30^{\circ}\right) \mathrm{in} \mathrm{fig.} 2$. The FYNES spectrum of the $\mathrm{CO}$ saturated surface, labeled $83 \mathrm{~K}$ in lig. 2 . exhibits the characteristic $\pi(288.5 \mathrm{eV})$ and $\sigma(306 \mathrm{eV})$ resonances of $(\%$ adsorbed on transition metals [14]. Heating the surface to $240 \mathrm{~K}$ to desorb the $\alpha_{1}$ adsorption state results in the spectrum labeled $240 \mathrm{~K}$ in lig. 2 . The desorption of the $\alpha_{1}$ state results in a small negative shift $(-0.3 \mathrm{eV})$ in the $\pi$ resonance and a somewhat larger shift $(-3.0 \mathrm{eV})$ in the o resonance. Heating the surface to $350 \mathrm{~K}$ desorbs the remaining $\alpha_{2}$ state and leaves only the $\alpha_{3}$ state ( $\pi$-honded $C O$ ) on the surface. It can be seen in fig. 2 that the FYNFS 


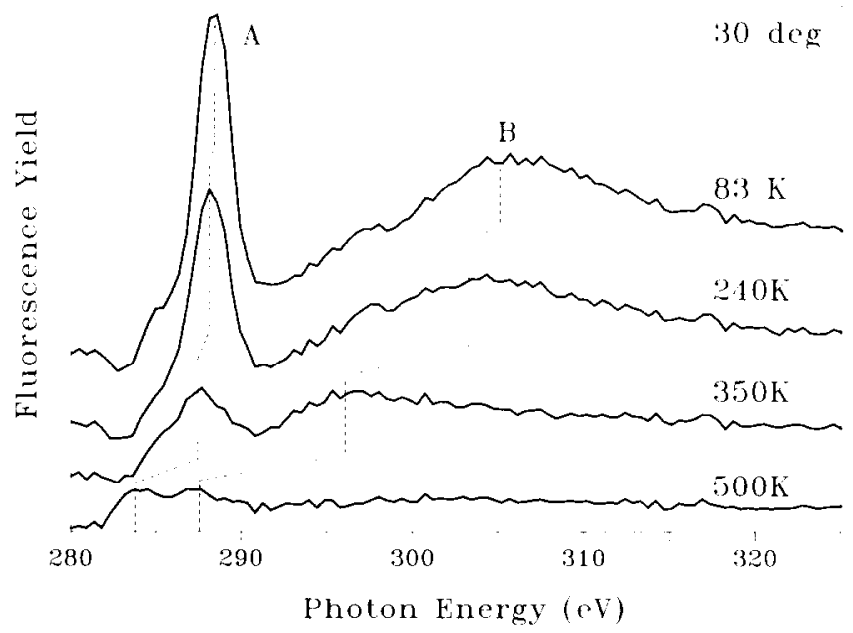

Fig. 2. FYNES spectra of the CO saturated $F e(100)$ surface as a function of annealing temperature. The temperature to the right of each spectrum indicates the annealing temperature. The angle of the electric field vector with respect to the surface normal was $30^{\circ}$. The A feature is the $\pi$ resonance and the $\mathrm{B}$ feature is the $\sigma$ resonance.

spectrum of $\alpha_{3}$ state (labeled $350 \mathrm{~K}$ ) is remarkably different from that of the other two molecular $\mathrm{CO}$ adsorption states. The $\pi$ resonance is weak and both the $\pi$ and $\sigma$ resonances exhibit a negative energy shift with respect to the $\alpha_{2}$ state. Heating the surface above the dissociation temperature $(T>440 \mathrm{~K})$ results in the FYNES spectrum labeled $500 \mathrm{~K}$ in fig. 2. This spectrum is hasically that of adsorbed atomic carbon on the Fe(100) surface. The $\pi$ resonance is replaced by an edge jump which appears at lower energy consistent with the large chemical shift in the $\mathrm{C} 1 \mathrm{~s}$ binding energy that is observed when $\mathrm{CO}$ dissociates [6].

The spectrum labeled $83 \mathrm{~K}$ in fig. 2 is that of a mixture of $\mathrm{CO}$ adsorption states. It contains signals from the $\alpha_{1}, \alpha_{2}$ and $\alpha_{3}$ states whereas the spectrum labcled $240 \mathrm{~K}$ contains only the $\alpha_{2}$ and $\alpha_{3}$ states. Subtraction of the $240 \mathrm{~K}$ spectrum from the $83 \mathrm{~K}$ spectrum yields the FYNES spectrum of the $\alpha_{1}$-state and subtraction of the $350 \mathrm{~K}$ spectrum from the $240 \mathrm{~K}$ spectrum yields the spectrum of the $\alpha_{2}$ state. The difference spectra obtained in this manner along the spectra of the $\alpha_{3}$ and $\beta$ (dissociated) $C O$ states are compared in fig. 3. The general trend in the spectra is a gradual shift in the position of the $\pi$ resonance to lower energy with a much more pronounced shift to lower energy in the position of the $\sigma$ resonance as the heat of adsorption increases. These shifts are a direct manifestation of the magnitude of the perturbation of the carbon-oxygen bond by the surface and will be discussed in more detail later.

A major application of NEXAFS is the determination of molecular orientation of adsorbed molecules. This approach has been particularly successful in 


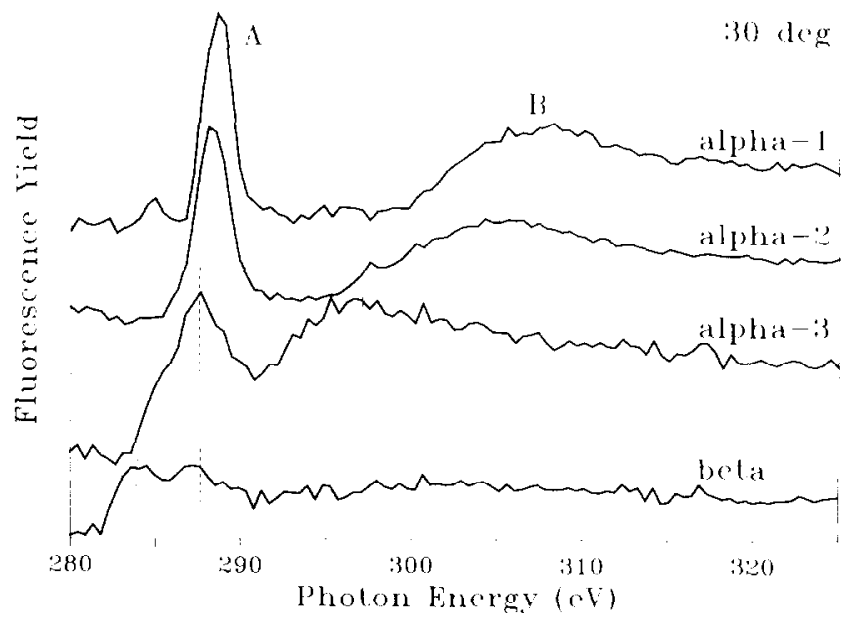

Fig. 3. FYNES spectra for the individual $\mathrm{CO}$ adsorption states taken with the electric field vector at $30^{\circ}$ from the surface normal. The $\alpha_{1}$ and $\alpha_{2}$ spectra were obtained by the difference technique.

the case of $\mathrm{CO}$ on transition metal surfaces due to the strong polarization dependence of the X-ray absorption features. The two X-ray absorption features of adsorbed $\mathrm{CO}$ arise from a transition of the carbon $1 \mathrm{~s}$ level ( $\sigma$-symmetry) to the partially occupied $2 \pi$ and the unoccupied $6 \sigma$ orbitals of adsorbed CO [16]. The dipole selection rule predicts that the $\alpha$ resonance will exhibit a maximum intensity when the electric field vector is perpendicular to the molecular axis, whereas the $\sigma$-resonance exhibits maximum intensity when the electric field vector is along the molecular axis. The experimental arrangement in the present study was such that the angle of incidence $\theta_{i}$ was also the angle of the electric field vector $\theta_{L}$ with respect to the surface normal. That is to say, where $\theta_{\mathrm{i}}=\theta_{E}=90^{\circ}$, the electric field vector is in the surface plane of the sample. In the case of $\mathrm{CO}$ bonded vertically with respect to the surface plane (the case for most transition metals) a maximum $\pi$ intensity and a minimum $\sigma$ intensity are observed at $\theta_{E}=90^{\circ}$. In the opposite polarization $\left(\theta_{E} \approx 0^{\circ}\right.$ ) the $\sigma$-resonance should reach a maximum and the $\pi$-resonance should vanish.

To probe the polarization dependence of each of the adsorbed $\mathrm{CO}$ states on this surface, FYNES spectra were collected at two additional angles of incident radiation. 'The data, after normalization by the incident X-ray flux, are presented in figs. 4-7 for each of the adsorption states.

The $\alpha_{1}$ and $\alpha_{2}$ states, shown in figs. 4 and 5 respectively, exhibit the classic response of $\mathrm{CO}$ adsorbed on transition metal surfaces. The $\pi$ resonance dominates at high angles of incidence $\left(\theta_{E}=90^{\circ}\right)$ but decreases rapidly as the electric field vector moves out of the surface plane towards the surface normal. 


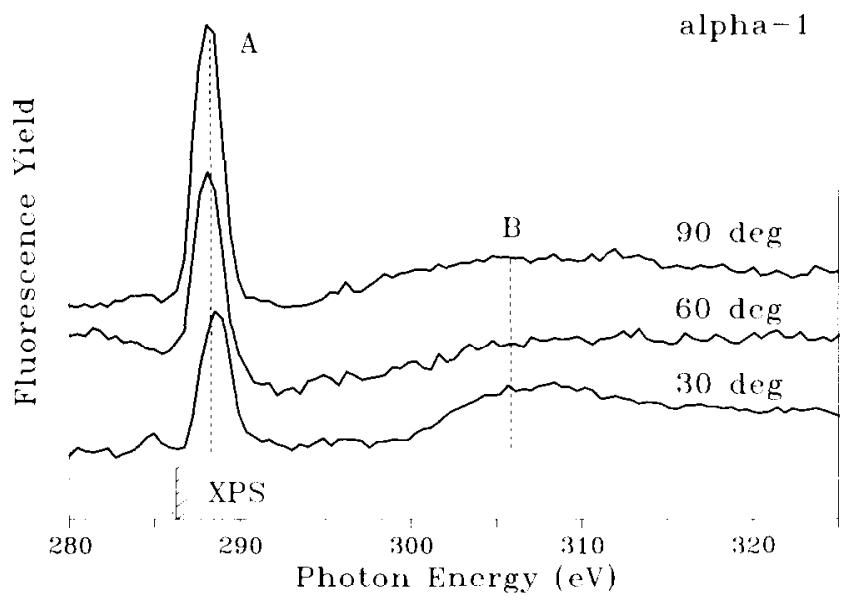

Fig. 4. FYNES spectra of the $\alpha_{1}$ state as a function of the angle of the electric field vector with respect to the surface normal.

In all likelihood, this is evidence for $\mathrm{CO}$ adsorbed with the carbon oxygen bond basically perpendicular to the surface plane. However, data at larger number of polarization angles are necessary to quantitatively determine any small differences in tilt angle that might exist between the two states. The $\alpha_{3}$ state, shown in fig. 6 , exhibits virtually no polarization dependence at the angles measured. These data are consistent with earlier data [9] which were rationalized by a molecular tilt angle close to the "magic angle" $\left(54.7^{\circ}\right)$ where

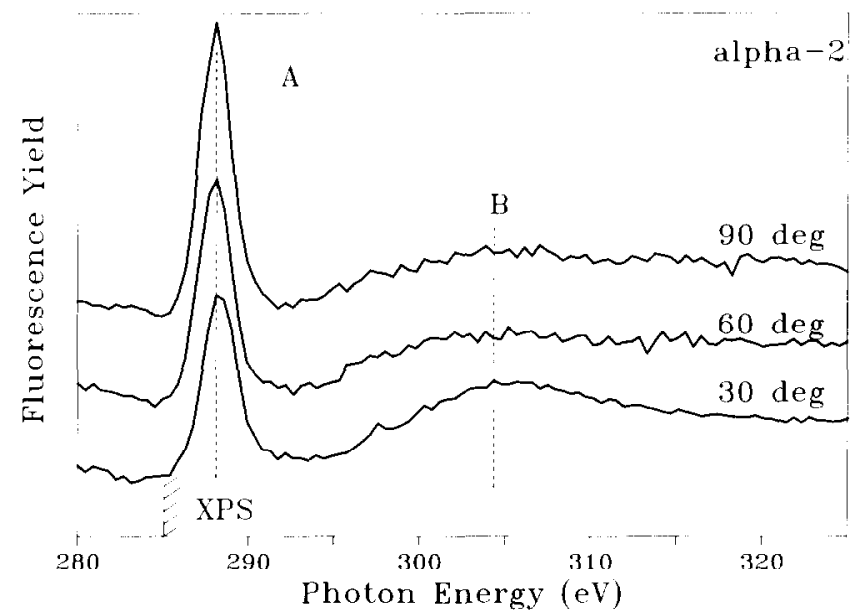

Fig. 5. FYNES spectra of the $\alpha_{2}$ CO adsorption state as a function of the angle to the electric field vector with respect to the surface normal. 


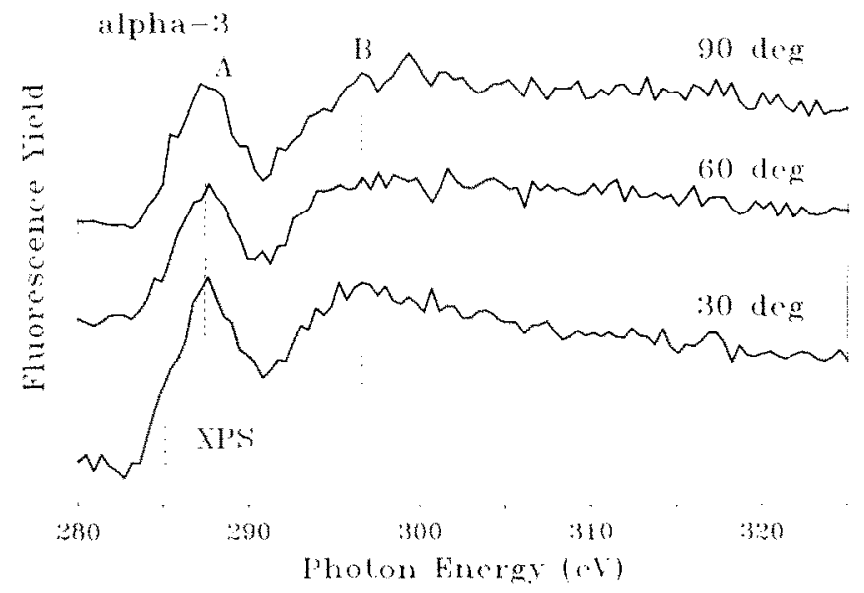

Fig. 6. FYNES spectra of the $\alpha_{3}$ CO adsorption state as a function of the angle of the wectric field vector with respect to the surface normal.

a lack of polarization is anticipated. However, this interpretation ignores possible rehybridization and a potential lowering of the symmetry of the parent molecular orbitals in this strongly perturbed $\mathrm{CO}$ adsorption state. The FYNES data obtained for the $\beta$ state (atomic carbon) were of low intensity and consequently poorer signal to noise as shown in fig. 7 . Although the signals are poor, there is a polarization dependence in the $\beta$ - $\mathrm{CO}$ signal which

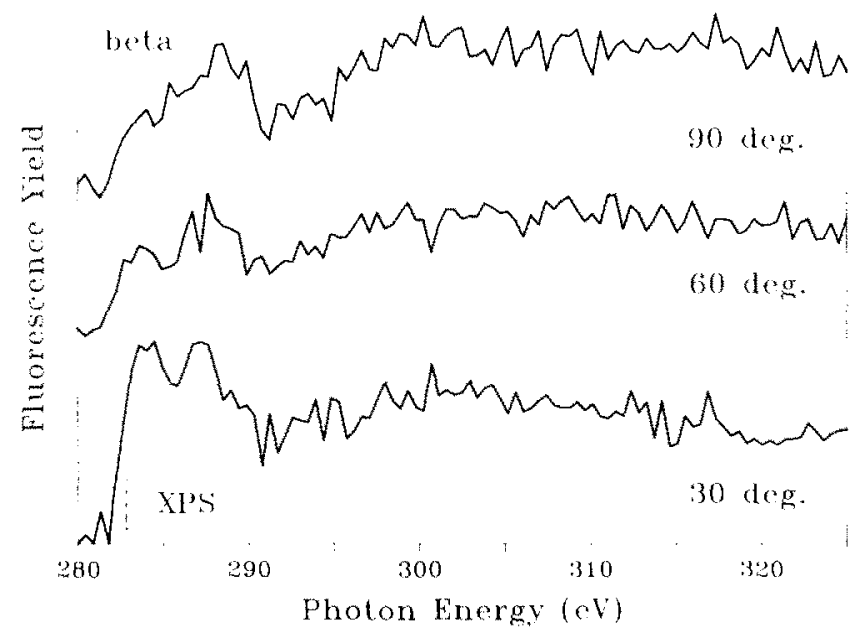

Fig. 7. FYNES spectra of the $\beta$ adsorption state (atomic carbon) as a function of the angle of the electric field vector with respect to the surface normal. 
is especially pronounced at edge. The origin of this effect is not clear but may be associated with local symmetry of the individual metal-carbon bonds in this system.

\section{Discussion}

In this manuscript we have presented near edge $X$-ray absorption spectra (obtained in the FYNES mode) for each of the three adsorption states of CO on Fe(100). The two adsorption states which are occupied at temperatures below $300 \mathrm{~K}$, the $\alpha_{1}$ and $\alpha_{2}$ states, exhibit near-edge spectra which are typical of $\mathrm{CO}$ chemisorbed on transition metal surfaces. A strong $\pi$ resonance is observed when the $\boldsymbol{E}$ vector is in the surface plane which decrease in intensity as the $\boldsymbol{E}$ vector moves towards the surface normal. The $\sigma$ resonance displays the opposite behavior with a maximum intensity observed with the $\boldsymbol{E}$ vector perpendicular to the surface plane. This type of polarization response signifies $\mathrm{CO}$ bound with molecular axis perpendicular to the macroscopic surface plane. These FYNES data are congruous with the normal carbon-oxygen vibrational frequencies (1950-2100 $\mathrm{cm}^{-1}$ ) and orbital energies (UPS data) associated with these adsorption states [7,8]. However, important differences exist in the FYNES spectra of these two adsorption states which reflect the degree to which the surface perturbs carbon-oxygen bond. For example, it can be seen in fig. 3 that the $\sigma$ resonance in the $\alpha_{2}$ spectrum is $2.9 \mathrm{eV}$ closer to the adsorption threshold than the $\sigma$-resonance in the $\alpha_{1}$ state. The variation in the $\alpha$ resonance position is known to be approximately linear with the $\mathrm{C}-\mathrm{O}$ bond length [17]. Using the relationship of Sette et al., $\Delta \sigma=a \Delta R$, where $a=32$ $\mathrm{eV} / \AA, \Delta \sigma$ is the $\sigma$ resonance shift and $\Delta R$ is the bond length change. The $\sigma$ resonance shift between the $\alpha_{1}$ and $\alpha_{2}$ states indicates that the $\mathrm{C}-\mathrm{O}$ bonds of molecules bound in the $\alpha_{2}$ state are $0.09 \AA$ longer than those bound in the $\alpha_{1}$ state. UPS spectra of these two states also exhibit different orbital energies for the $5 \sigma$ and $1 \pi$ molecular orbitals [6]. These UPS data were interpreted in terms of weakly bound, almost physisorbed. $\alpha_{1}$ state with the $\alpha_{2}$ slate being a more conventional chemisorbed configuration. The present data supports that interpretation. A shorter $\mathrm{C}-\mathrm{O}$ bond, approaching the gas phase value, is expected in the physisorbed state whereas back-bonding will weaken the $\mathrm{C}-\mathrm{O}$ bond in the chemisorbed state [18].

The FYNES spectrum of the $\alpha_{3}$ state is distinct from near-edge spectra of conventionally bound $\mathrm{CO}$. The $\pi$ resonance is shifted down in energy very close to the absorption threshold (fig. 3) and the $\sigma$ intensity is extremely weak and broad in all polarizations (fig. 6). Analyzing these spectra within the framework of the unoccupied orbitals of the parent molecule yields a $\mathrm{C}-\mathrm{O}$ bond length of $1.43 \AA$ and a molecular tilt of approximately $54.7^{\circ}$ (so-called "magic angle"). In this scheme, the weak $\pi$ resonance is interpreted in terms 
of extensive occupation of $2 \pi^{*}$ which lowers the transition probability for the $\mathrm{X}$-ray absorption process. The unusually large width of $\pi$ resonance is associated with a lifting of the degeneracy of the $\pi$ orbitals via a direct interaction with the surface. This type of line broadening has been observed in the case of $\mathrm{CN}$ parallel bonded to $\mathrm{Pd}(111)$ [19]. In fact. a splitting of the $\pi$ resonance of into two distinct peaks due to the interaction of the $\pi$ orbitals with the surface (one set of $\pi$ orbitals interacting, one set non-interacting) is reported for that case.

Although it is possible to generate this self-consistent model for the near-edge spectrum of the $\alpha_{3}$ state in terms of unmodified molecular orbitals of $\mathrm{CO}$, a note of caution must be raised. The UPS spectra of $\pi$-bonded $\mathrm{CO}$ on both the $\mathrm{Fe}(100)$ and $\mathrm{Cr}(111)$ surfaces clearly show that the uccupied nolecular orbitals of the $\pi$-bonded $\mathrm{CO}$ are extensively rehybridized with respect $t$ conventionally bound $\mathrm{CO}$. The extensive reorganization of the carbon-..oxygen bond in the $\alpha_{3}$ state brings into question the assignment of the " $\pi$ " and " $\sigma$ " resonances in the spectra of the $\alpha_{3}$ state. The unusual position and polarization response of these features in the near-edge spectrum of the $\alpha_{3}$ state may reflect a lowering of the carbon-oxygen bond order. In this case, it would not be appropriate to interpret the spectral feature in terms of $\pi$ and $\sigma$ resonances of $\mathrm{CO}$. It is interesting in this respect that the near-edge spectrum of the $\alpha_{2}$ state is reminiscent of the spectra of adsorbed methoxy [20] which contains a carbon-oxygen single bond. A lowering of the $\mathrm{CO}$ bond order from three 10 one would also explain the exceptionally low stretching frequency of the carbon-oxygen bond as well as the UPS data. Theoretical input in the form of orbital energies and symmetry assignments are required to differentiate between this two possible interpretations of the near-edge spectrum of the $\alpha$; state. The exact orientation of the carbon-oxygen bond with respect to the surface, therefore, remains uncertain.

\section{Acknowledgements}

The authors would like to thank the members of the Exxon PRT team for the use of the beamline and their help in setting up the experiment. One of us D.D. would like to acknowledge the support of the NSLS/HFBR Faculty and Student Support Program. We would also like to thank the Rolling Stones for supplying the sound track for this experiment.

\section{References}

(1) D.W. Moon. S.L. Bernasek, J.P. Lu. J.L. Gland and D.J. Dwyer, Surface Sci. 184 (1987)90.

[2] K. Kishi and M.W. Roberts, J. Chem. Soc. Faraday Trans. I. 71 (1975) 1715. 
[3] T.N. Rhodin and C.F. Brucker, Solid State Commun. 23 (1977) 275.

[4] C.R. Brundle, IBM J. Res. Develop 22 (1978) 235.

[5] J. Benziger and R.J. Madix, Surface Sci. 94 (1980) 119.

[6] S.D. Cameron and D.J. Dwyer, Langmuir 4 (1988) 282.

[7] D.W. Moon. S.L. Bernasek, D.J. Dwyer and J.L. Gland, J. Am. Chem. Soc. 107 (1985) 4363.

[8] C. Benndorf, B. Kruger and F. Thieme, Surface Sci. 163 (1985) L675.

[9] D.W. Moon, S. Cameron, F. Zaera, W. Eberhardt, R. Carr, S.L. Bernasek, J.L. Gland and D.J. Dwyer, Surface Sci. 180 (1987) L123.

[10] C. Benndorf. B. Nieber and B. Kruger, Surface Sci. 177 (1986) L907.

[11] C.P. Horowitz, E.M. Holt and D.F. Shriver, J. Am. Chem. Soc. 107 (1985) 281.

[12] S.P. Mehandru and A.B. Anderson. Surface Sci. 201 (1988) 345.

[13] D.A. Fischer, U. Dobler, D. Arvanitis, L. Wenzel. K. Baberschke and J. Stohr, Surface Sci. 177 (1986) 144.

[14] F. 7.aera, D.A. Fischer, S. Shen and II Gland, Surface Sci. 194 (1988) 205

[15] D.A. Fischer, J. Colbert and J.L. Gland, Rev. Sci. Instr.. in press.

[16] J. Stohr and R. Jaeger, Phys. Rev. B 26 (1982) 4111.

[17] F. Sette, J. Stohr and A.P. Hitchcock, Chem. Phys. Letters 110 (1984) 517.

[18] S. Sung and R. Hoffmann, J. Am. Chem. Soc. 107 (1985) 578.

[19] J. Somers, M.E. Kordesch. Th. Linder. H. Conrad, A.M. Bradshaw and G.P. Williams. Surface Sci. 188 (1987) L693.

[20] J. Stohr and D.A. Outka, J. Vacuum Sci. Technol. A 5 (1987) 919. 